\title{
Die Leichenschau
}

Clara-Sophie Schwarz, Isabelle Gutmann, Roman Bux, Kathrin Yen

\begin{abstract}
Wenn in Deutschland ein Mensch stirbt, ist eine Leichenschau Pflicht. Die Untersuchung des Verstorbenen dient u. a. dazu, den Tod festzustellen und Aufschluss über die Ursachen und näheren Umstände des Todes zu bekommen. Wie Sie eine Leichenschau lege artis durchführen und worauf Sie beim Betreten des Fundorts und der Untersuchung besonders achten müssen, erfahren Sie in diesem Beitrag.
\end{abstract}

\section{Erste Schritte am Fundort}

An die eigene Sicherheit denken | Rückt der Leichenschauarzt zu einem ihm unbekannten Ort aus, ist es sinnvoll, wenn er sich zunächst einen Überblick über die Situation verschafft und gegebenenfalls Maßnahmen der Eigensicherung trifft. Dies gilt insbesondere bei

- Mehrleichenfunden,

- Tierkadavern in der Nähe des Leichnams,

- verschlossenen Garagen, Silos usw. (Gefahr der Gasvergiftung).

Für optimale Bedingungen sorgen | Vor Ort sind Bedingungen zu schaffen, die eine sorgfältige Durchführung der Leichenschau ermöglichen. Hierzu gehört vor allem eine angemessene Beleuchtung. Es lohnt sich daher, neben zwei Pinzetten zum Ektropionieren der Augenlider ( Abb. 1) und Handschuhen auch eine Taschenlampe mit sich zu führen. Bei unzureichender Beleuchtung muss die Leichenschau an einem anderen Ort mit besseren Bedingungen wiederholt bzw. nach Feststellen des Todes fortgeführt werden.

Abb. 1 Die Augenlider werden am besten mit Pinzetten ektropioniert. In diesem Fall sind Stauungsblutungen in den Konjunktiven zu erkennen. Diese können ein Hinweis auf einen Angriff gegen den Hals (z. B. Würgen) sein.

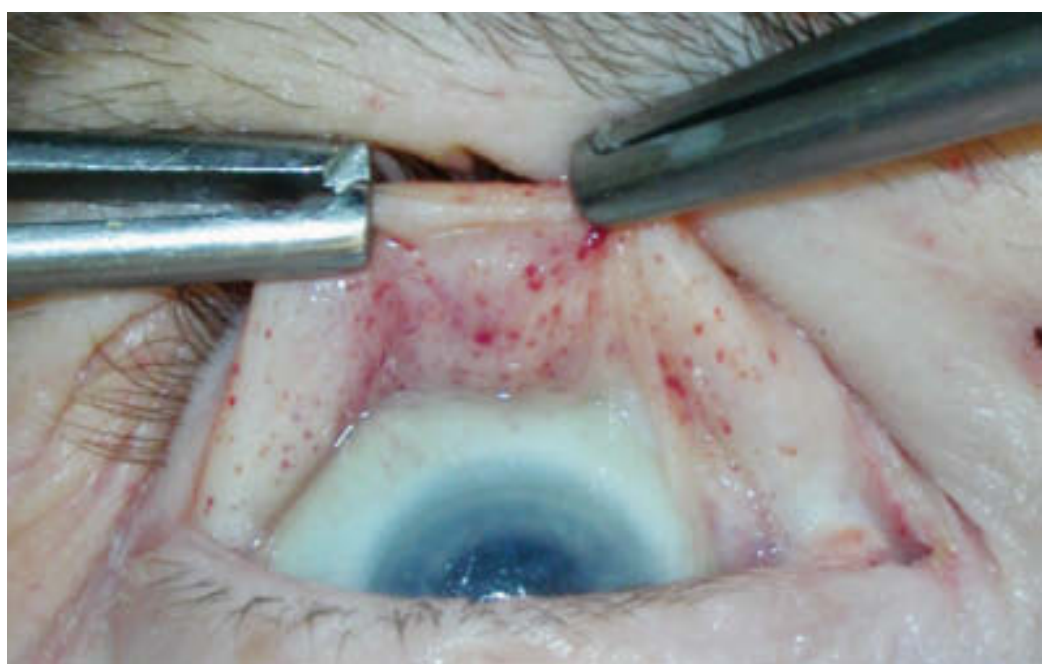

\section{Todesfeststellung}

Sichere Todeszeichen I Am Beginn der Leichenschau steht zunächst die Feststellung des Todes. Zur abschließenden Todesfeststellung genügt es, wenn eines der sicheren Todeszeichen vorliegt. Die sicheren Todeszeichen sind:

- Totenflecke

- Totenstarre

- Fäulnis

Daneben besteht die Möglichkeit, den Tod anhand nicht mit dem Leben vereinbarer Verletzungen festzustellen. Dies ist zulässig, wenn zum Beispiel eine Dekapitation oder eine Durchtrennung des Rumpfes vorliegt. Andernfalls ist hier Zurückhaltung geboten. Ein schweres Polytrauma, Verlust von Extremitäten oder Ähnliches genügt nicht zur Todesfeststellung.

Unsichere Todeszeichen I Unsichere Todeszeichen eignen sich nicht zur Todesfeststellung und können einen reanimationspflichtigen Zustand anzeigen. Es handelt sich im Wesentlichen um die Nichtfeststellbarkeit von Lebenszeichen wie Puls und Atmung sowie lichtstarre Pupillen und eine niedrige Körpertemperatur. Fehlen sichere Todeszeichen, sollte eine leblose Person erst als tot betrachtet werden,

- wenn eine kardiopulmonale Reanimation erfolglos bleibt oder aussichtlos ist oder

- wenn bei erhaltenem Kreislauf die Hirntoddiagnostik abgeschlossen ist.

Wird aufgrund einer Patientenverfügung auf eine Reanimation verzichtet, dann ist mit dem Ausfüllen der Todesbescheinigung so lange zu warten, bis die ersten sicheren Todeszeichen eintreten.

Die Polizei ist zu informieren bei

- unbekannter Leiche,

- Anhaltspunkten für einen nicht-natürlichen Tod (Unfall, Suizid, Tötung),

- ungeklärtem Tod (wenn diese Möglichkeit im jeweiligen Bundesland vorgesehen ist). 

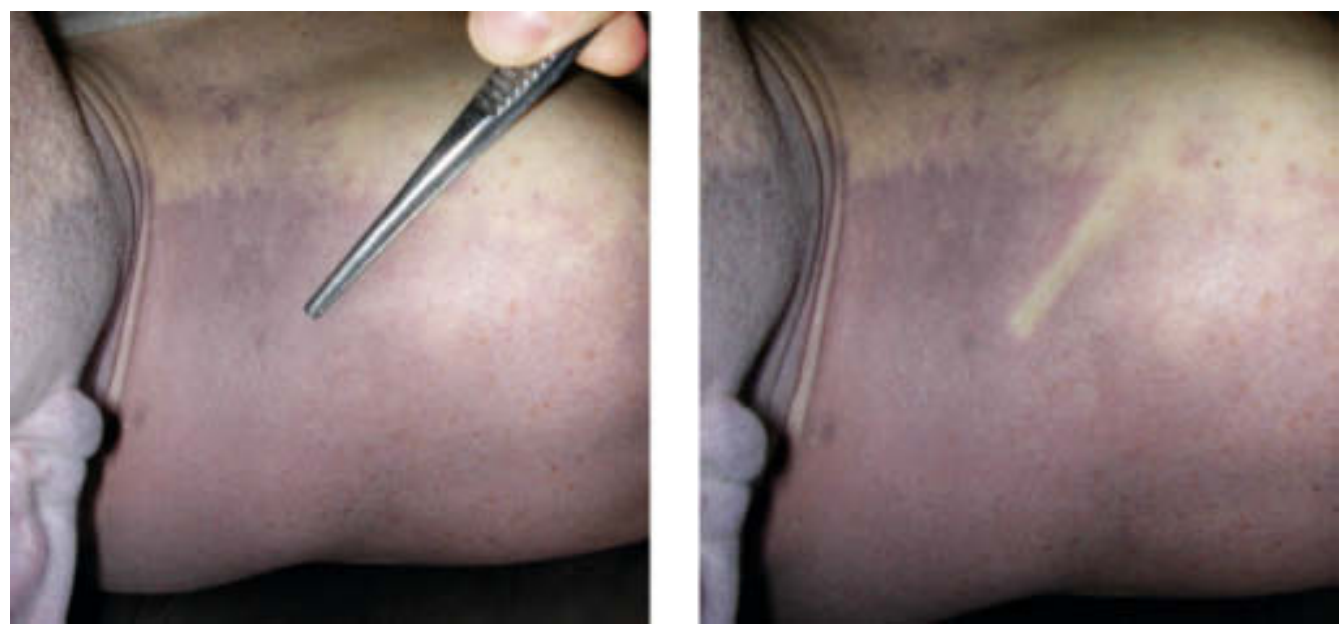

\section{Totenflecke}

Das erste sichere Todeszeichen I Infolge der Senkungsblutfülle von Hautgefäßen in den abhängigen Körperpartien bilden sich ca. 15-20 Minuten nach dem Kreislaufstillstand die Totenflecke (Livores) - das erste sichere Todeszeichen. Sie erscheinen zunächst als fleckige Verfärbungen, die mit der Zeit konfluieren. Aufgrund der postmortalen Sauerstoffentsättigung des Bluts nehmen sie in der Regel einen blau-lividen Farbton an.

Bei Rückenlage des Verstorbenen ist im Regelfall eine Ausprägung bis in die mittlere Axillarlinie reichend zu erwarten.

Umlagerbarkeit und Wegdrückbarkeit | Mit zunehmender Liegezeit werden flüssige Blutbestandteile aus den Gefäßen herausgepresst. So konzentrieren sich feste Blutbestandteile in den Gefäßen und schließlich - bei zunehmender Durchlässigkeit der Gefäßwände - tritt Blutfarbstoff in das Gewebe aus. Diese Vorgänge erklären die über die Zeit abnehmende

- Umlagerbarkeit (vollständige oder teilweise Neubildung nach Lageänderung entsprechend der neuen Lage) und

- Wegdrückbarkeit der Totenflecke (Abblassen auf Fingerkuppendruck über einem Knochenpunkt, z. B. dem Schulterblatt [ Abb. 2]).

Diese Phänomene sind bis ca. 10 Stunden nach dem Tod nachweisbar.

Verteilung I Die Verteilung der Totenflecke am Körper ist kriminalistisch bedeutsam, denn eine Ausprägung der Totenflecke, die nicht zur Auffindesituation passt, deutet auf eine Lageänderung nach dem Tod hin. Stellt man hier Diskrepanzen fest, gibt eine Befragung der Leichenfinder häufig Aufschluss. Es kommt zum Beispiel vor, dass Angehörige einen Leichnam von der Bauch- in die Rückenlage drehen, damit eine Verabschiedung möglich ist. Bleiben Ungereimtheiten, sollten Sie dies der Polizei melden.
Ausprägung und Farbe I Eine geringe Ausprägung der Totenflecke kann auf eine chronische oder akute Anämie hinweisen. Abweichende FärbunTotenflecke bei einer Kohlenmonoxidintoxikation wegen der hohen Sättigung des Hämoglobins mit Kohlenmonoxid typischerweise einheitlich hell$\operatorname{rot}(\triangleright$ Abb. 3).

\section{Differenzialdiagnostisch können hellrote}

Totenflecke auch dafür sprechen, dass der Leichnam einer niedrigen Umgebungstemperatur ausgesetzt war: Kälte begünstigt die Diffusion von Sauerstoff in die Gefäße und seine Bindung an Hämoglobin.

Auf die Finger schauen | Es empfiehlt sich, die Farbe der Nagelbetten zu betrachten - sofern diese im Totenflecksystem gelegen sind. Ist die hellrote Farbe der Totenflecke kältebedingt, werden die Nagelbetten trotzdem eine livide Färbung aufweisen, da die Hornsubstanz das Eintreten von Sauerstoff aus der Umwelt verhindert. Bei einer Kohlenmonoxidintoxikation (inneres Ersticken) hingegen werden auch die Nagelbetten eine hellrote Farbe zeigen.

Flecke richtig unterscheiden | Ein genaues Hinschauen verlangt u.U. auch die Unterscheidung

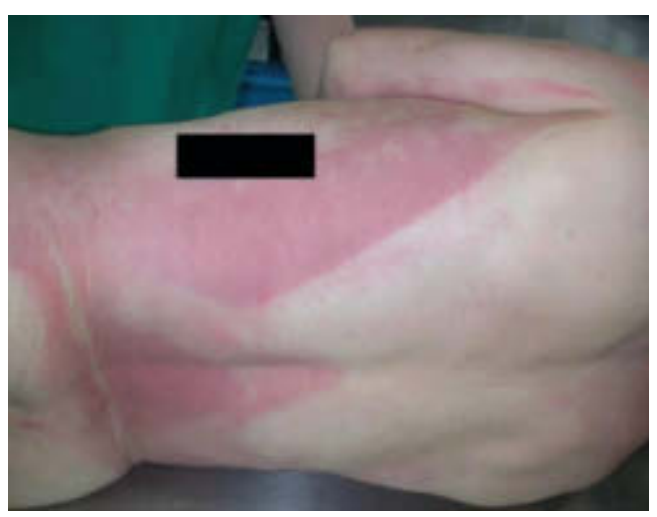
gen sind bei Vergiftungen zu beobachten. So sind
Abb. 2 Bis ca. 10 Stunden nach dem Tod lassen sich die Totenflecke wegdrücken.
Abb. 3 Hellrote Totenflecke bei Kohlenmonoxidvergiftung. 
Abb. 4 Totenstarre. Auffallend ist die Fehlstellung des Handgelenks. Die Obduktion ergab eine Radiusfraktur.

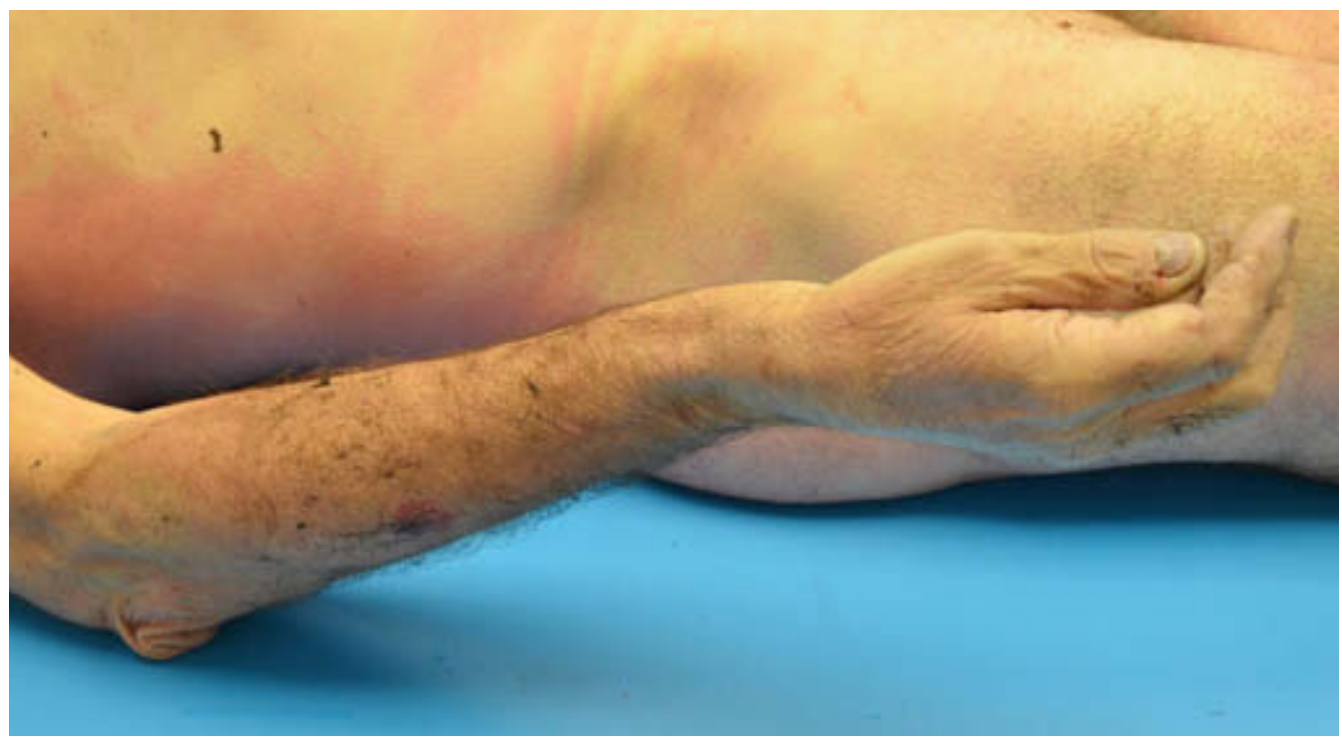

zwischen Totenflecken und Hautunterblutungen. Um Hämatome zu erkennen, ist es hilfreich, beim Betasten auf Schwellungen und Verhärtungen des Gewebes zu achten und nach Hautschürfungen zu suchen.

Da sich der Blutfarbstoff bei Hämatomen außerhalb der Gefäße befindet, ist ein Wegdrücken wie bei den (frühen) Leichenflecken nicht möglich.

Auch Totenfleckberstungsblutungen (Vibices) und die stecknadelkopfgroßen Stauungsblutungen sind nicht leicht auseinanderzuhalten. Letztere treten häufig bei oberer Einflussstauung (z. B. bei einem Angriff gegen den Hals) auf ( $\downarrow$ Abb. 1).

Vibices kommen im Unterschied zu diesen petechialen Stauungsblutungen ausschließlich im Totenfleckbereich vor.

Schwer abzugrenzen sind Totenflecke mitunter bei Leichen dunkelhäutiger Personen. Hier kann

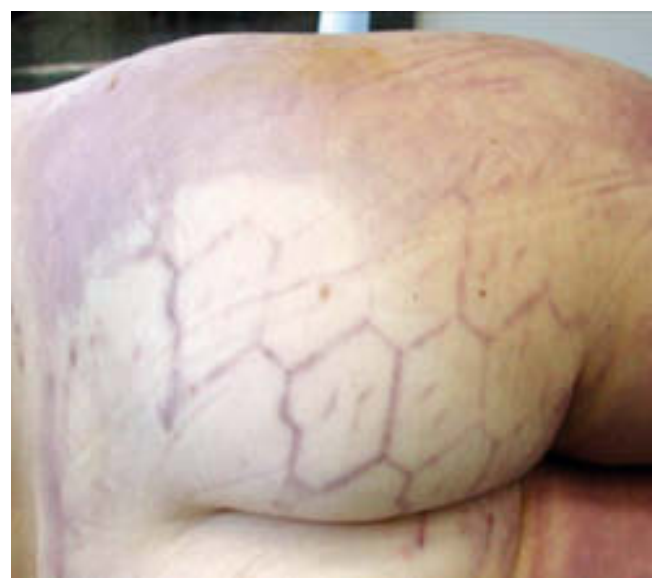

es helfen, die typischen Stellen (z.B. Nacken bei Rückenlage) mit Fingerkuppen- oder -nageldruck zu untersuchen und auf die auftretenden Farbunterschiede zu achten.

\section{Totenstarre}

Zeitlicher Verlauf | Weil die ATP-Konzentration in den Muskeln postmortal sinkt, kommt es ca. 2-4 Stunden nach dem Tod zur spürbaren Ausbildung der Totenstarre (Rigor mortis [ $\mathbf{A b b} .4]$ ), sofern Körpers oder hitzebedingte Schrumpfung und Denaturierung der Muskulatur verhindert wird. Der Prozess läuft nicht in allen Muskeln oder in allen Anteilen eines Muskels gleichzeitig ab. So ist in Muskeln, die vor dem Tod stärker beansprucht wurden, aufgrund der geringeren ATP-Reserven eine schnellere und kräftigere Ausbildung zu erintervalls von 8 Stunden (in einigen Fällen bis 19 Stunden) gebrochen, ist eine Neubildung möglich. Autolysevorgänge führen nach wenigen Tagen zur endgültigen Lösung der Starre. Diese Vorgänge sind stark temperaturabhängig.

Verwechslungsgefahr I Es besteht die Gefahr, dass Kältestarre oder Kontrakturen mit einer Totenstarre verwechselt werden. Zur Prüfung der Totenstarre empfiehlt es sich, Kiefergelenk und große und kleinere Gelenke im Seitenvergleich passiv zu bewegen, um etwaige Widerstände und deren Ausprägung in mehr als nur in einem Gelenk beurteilen zu können.

\section{Vertrocknungen}

Hervortreten kleinster Befunde I Zu den frühen Leichenerscheinungen gehört auch das Vertrocknen von Haut und Schleimhäuten, insbedies nicht durch ausgedehnte Zerstörung des warten. Wird die Starre innerhalb eines Zeit- 
sondere im Bereich der Konjunktiven und Hornhäute bei unvollständigem Lidschluss, der Lippen, Akren, großen Schamlippen und des Hodensacks. Auch oberflächliche Hautschürfungen mit freiliegenden tieferen Hautschichten können nach dem Tod gelb-bräunlich vertrocknen und sich lederartig verhärten. Kleinste Befunde können durch die Vertrocknung innerhalb kurzer Zeit deutlich hervortreten, zum Beispiel periorale Verletzungen nach einem Angriff gegen die Atemöffnungen.

\section{Fäulnis}

Ursachen \& Erscheinungsbild | Fäulnis wird durch die Ausbreitung und Stoffwechselaktivität vorwiegend anaerober Bakterien hervorgerufen. Diese besiedeln beim Lebenden üblicherweise den Darm und verteilen sich von dort aus, nachdem die Barrieren des lebenden Organismus zusammengebrochen sind. Wie schnell das geschieht, ist von der Umgebungstemperatur und anderen Einflussfaktoren abhängig.

Oft tritt als erstes Anzeichen der Fäulnis eine Grünverfärbung des Unterbauchs auf. Diese kann bei flüchtiger Inspektion mit älteren Hämatomen verwechselt werden.

Im Weiteren breiten sich Fäulnisbakterien bevorzugt entlang venöser Blutgefäße aus, sodass es zu einem „Durchschlagen“ des Venennetzes kommt. Fäulnis zeigt sich

- an einer typischen Verfärbung,

- Blasenbildung und

- Ablösung der Haut.

Daneben führen Fäulnisgase zu einer Blähung und Aufdunsung des Leichnams. Fäulnisflüssigkeit mit einer schmutzig braun-rötlichen Farbe kann aus Körperöffnungen und eröffneten Fäulnisblasen austreten, wobei differenzialdiagnostisch an ablaufendes Blut zu denken ist. Fliegeneier werden häufig in die Augenwinkel und Gesichtsöffnungen sowie in bestehende offene Wunden abgelegt. Eine Körperstelle mit auffallend starker Madenbesiedlung kann daher eine Verletzung verbergen und bedarf besonderer Aufmerksamkeit.

Identifikation fäulnisveränderter Leichen | Vom Versuch einer visuellen Identifizierung fortgeschritten fäulnisveränderter Leichen sollte abgesehen werden, da es leicht zu Fehleinschätzungen kommt. Die fäulnisbedingte Gasblähung kann einen unzutreffenden Eindruck von Körpergewicht und Konstitution erwecken. Dunsung und Verfärbung des Gesichts können die Gesichtszüge völlig verändern. Deshalb müssen Sie den Angaben Dritter mit Misstrauen begegnen.
Wenn sich der leichenschauende Arzt über die Identität des Toten nicht sicher ist, sollte er sich keinesfalls unter Druck setzen lassen, die Todesbescheinigung für eine bestimmte Person (z. B. den Bewohner einer Wohnung, in der der Leichnam aufgefunden wurde) auszustellen.

\section{Weiterer Ablauf der Leichenschau}

Leichnam entkleiden | Der vollständig entkleidete Leichnam ist gründlich zu inspizieren. Sehr hilfreich ist es, den Ablauf der Untersuchung immer gleich zu gestalten - z. B. immer von oben nach unten $\mathrm{zu}$ arbeiten. Dabei sind stets auch behaarte Kopfhaut, Körpervorder- und -rückseite sowie die Körperöffnungen zu untersuchen. Eine lückenlose Inspektion ist nur nach vollständigem Entkleiden und Entfernen von Pflastern und Verbänden zu erreichen ( $\bullet$ Abb. 5 und $\mathbf{6}$ ). Das Skelett sollte auf Fehlbeweglichkeiten überprüft werden. Insbesondere die ektropionierten Lidbindehäute, die Haut hinter den Ohren und die Mundvorhofschleimhaut ( $\triangleright$ Abb. 7) müssen gezielt auf Stauungsblutungen hin untersucht werden.

Erschwerte Bedingungen | Für den leichenschauenden Arzt können die Bedingungen für die Untersuchung erschwert sein, z. B. wenn

- keine Hilfsperson zum Entkleiden und Drehen des Leichnams verfügbar ist,

- Angehörige anwesend sind,

- der Fundort unübersichtlich ist.

Bei Fragen oder Unsicherheiten besteht die Möglichkeit, die zuständige Rechtsmedizin zu kontaktieren.

Todeszeitschätzung I Der Gesetzgeber sieht vor, dass sich der leichenschauende Arzt zur Todeszeit äußert. Zur Eingrenzung kann die Phänomenologie der sicheren Todeszeichen ( $\bullet$ Tab. 1 ) herangezogen werden.

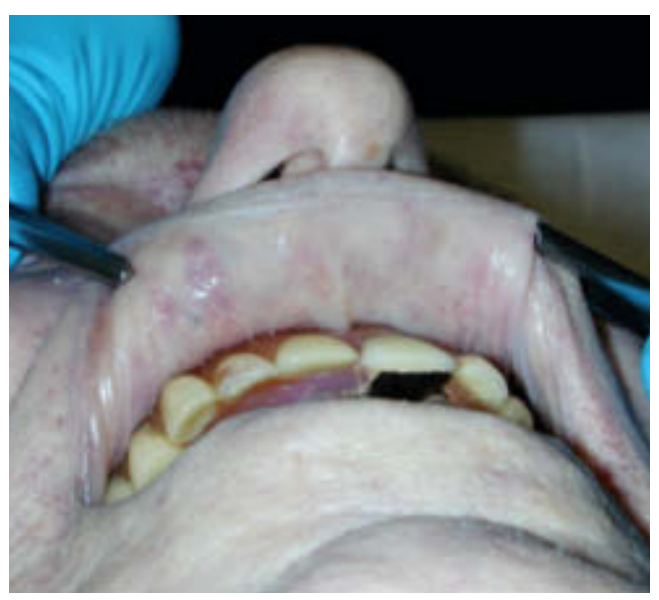

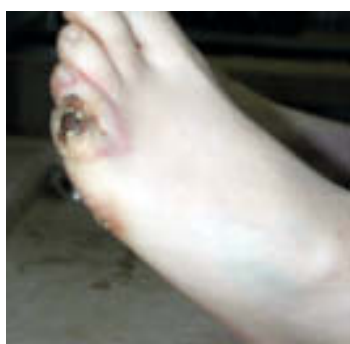

Abb. 6 Im Fall des plötzlichen Todes einer Bäuerin bei der Arbeit wurde zunächst ein natürlicher Tod bescheinigt. Erst bei der zweiten Leichenschau wurde die Leiche entkleidet. Es fand sich eine Strommarke am Fuß, sodass unter Berücksichtigung der Fallumstände von einem Unfalltod auszugehen war und die Angehörigen der Verstorbenen Ansprüche bei einer Unfallversicherung geltend machen konnten.

Abb. 7 Stauungsblutungen in der Mundvorhofschleimhaut. In diesem Fall führte Erhängen zum Tod. 


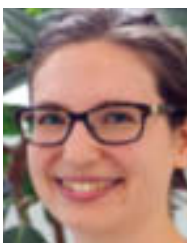

Dr. med.

Clara-Sophie Schwarz ist wissenschaftliche Mitarbeiterin am Institut für Rechtsmedizin und Verkehrsmedizin an der Uniklinik Heidelberg. Clara-Sophie.Schwarz@med. uni-heidelberg.de

\section{Dr. med.}

Anna Isabelle Gutmann ist wissenschaftliche Mitarbeiterin am Institut für Rechtsmedizin und Verkehrsmedizin an der Uniklinik Heidelberg. isabelle.gutmann@med. uni-heidelberg.de

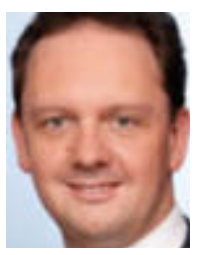

Dr. med. Roman Bux, MBA ist Oberarzt am Institut für Rechtsmedizin und Verkehrsmedizin an der Uniklinik Heidelberg. roman.bux@med. uni-heidelberg.de

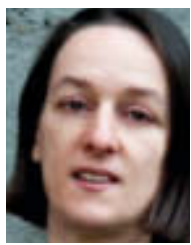

Prof. Dr. med. univ. Kathrin Yen ist Ärztliche Direktorin am Institut für Rechtsmedizin und Verkehrsmedizin an der Uniklinik Heidelberg. kathrin.yen@med. uni-heidelberg.de

\section{Interessenkonflikt}

Die Autoren geben an, dass kein Interessenkonflikt besteht.

DOI 10.1055/s-0041-103283

Dtsch Med Wochenschr 2015; 140: 1148-1152

(c) Georg Thieme Verlag KG . Stuttgart · New York .

ISSN 0012-0472

\author{
Livores \\ Auftreten ca. 20 min nach dem Tod \\ Konfluktion nach ca. 1-2 h
}

Vollständiges Abblassen auf Fingerkuppendruck, Umlagerbarkeit bis ca. $10 \mathrm{~h}$ nach dem Tod

Tab. 1 Todeszeitschätzung anhand der Leichenbefunde (Livores und Rigor mortis).

Hilfreich ist es, zusätzlich Informationen aus dem Umfeld, zum Beispiel über den Zeitpunkt des letzten Kontakts, mit einzubeziehen und ggf. ihre Plausibilität anhand der Leichenbefunde zu prüfen.

Eine Todeszeitschätzung basierend auf den am Leichnam erhobenen Befunden kann schwierig sein. Ist in speziellen Fällen eine genauere Aussage erforderlich, so lassen sich zusätzliche Erkenntnisse im Rahmen rechtsmedizinischer Fundortarbeit gewinnen. Je nach Fallumständen können die tiefe Rektal- und die Umgebungstemperatur (unter Berücksichtigung der Bedingungen am Fundort) sowie die supravitalen Reaktionen dem Rechtsmediziner helfen, die Todeszeit weiter einzugrenzen. Supravitale Reaktionen beruhen auf der über den Individualtod hinausgehenden Reagibilität einzelner Gewebe auf äußere Reize, die liegezeitabhängig ist. Dazu zählen Muskelkontraktionen nach mechanischer und elektrischer Reizung und Pupillenreaktionen auf Pharmaka.

Umgebung des Leichnams I Die Umgebung des Leichnams hält oft wichtige Hinweise auf den Geschehensablauf (z.B. Medikamente, Fixerutensilien, Abschiedsbriefe, erbrochenes Hämatin) bereit. Beobachtungen am Fundort können sich als außerordentlich wertvoll erweisen und sind nachträglich oft nicht mehr erhältlich. Wenn Sie die Umgebung des Leichnams in Augenschein nehmen, müssen Sie zu jedem Zeitpunkt auf Selbstschutz achten. Beim blinden Griff in Taschen oder Abfalleimer besteht die Gefahr des Stechens an spitzen Gegenständen wie Spritzennadeln. Auch Stromquellen können eine Gefahr darstellen. Sind Polizeibeamte vor Ort, sollten Sie sich mit ihnen vor dem Hintergrund einer möglicherweise noch durchzuführenden Spurensicherung hinsichtlich des Vorgehens genau absprechen.

\section{Rigor mortis}

Beginn ca. 2-4h nach dem Tod

Wiedereintritt nach Brechen bis ca. $8 \mathrm{~h}$ (max. 19h) nach dem Tod

Beginn der Lösung ab ca. 2-3 Tage nach dem Tod (stark temperaturabhängig)
Konsequenz für Klinik und Praxis

- Eigenschutz und Umgebungssicherung stehen an erster Stelle.

- Todesfeststellung:

> Totenflecke

- Totenstarre

> Fäulnis

nicht mit dem Leben vereinbare Verletzungen

- abgeschlossene Hirntoddiagnostik

- Achten Sie auf Hinweise aus der Auffindesituation.

- Die Inspektion erfolgt am vollständig entkleideten Leichnam. Achten Sie auf Hinweise wie Hämatome, Einstichstellen, Verletzungen usw.

- Inspizieren Sie auch die Körperöffnungen.

- Prüfen Sie das Skelett auf Fehlbeweglichkeiten.

- Holen Sie sich Auskünfte des behandelnden Arztes und von Anwesenden ein.

- Füllen Sie die Todesbescheinigung vollständig aus.

- Ergeben sich zu irgendeinem Zeitpunkt Anhaltspunkte für einen nicht-natürlichen Tod, sollten Sie sofort abbrechen und die Polizei informieren!

\section{Literatur}

1 Anders S, Kunz M, Gehl A et al. Estimation of the time since death - reconsidering the re-establishment of rigor mortis. Int J Legal Med 2013; 127: 127-130

2 Grassberger M, Schmid H. Todesermittlung. Befundaufnahme \& Spurensicherung; ein praktischer Leitfaden für Polizei, Juristen und Ärzte. Wien u. a: Springer; 2009

3 Madea B. Die ärztliche Leichenschau. Rechtsgrundlagen, praktische Durchführung, Problemlösungen. 2., aktualisierte Aufl. Heidelberg: Springer; 2006

4 Madea B. Praxis Rechtsmedizin. Befunderhebung, Rekonstruktion, Begutachtung. 2., aktualisierte Aufl. Berlin u. a: Springer; 2007 